\title{
Synthesis and Biological Activity of Some Novel Phenyl Pyrazoline Derivatives
}

\author{
SHAILESH H SHAH* and PANKAJ S PATEL
}

*Department of Chemistry, Patel JBR Arts, Patel AMR Commerce \& Patel JDKD Science College, Borsad \& JJT-University, Rajasthan, India

Department of Chemistry, Sheth LH Science College, Mansa, India

shailchem@yahoo.com

Received 26 June 2012 / Accepted 8 July 2012

\begin{abstract}
A new series of 4-(4-hydroxyphenyl)-3-chloro-1-\{4-[5-(substituted phenyl)-1-phenyl- 4,5dihydro-pyrazol-3-yl]phenyl $\}$ azetidin-2-one were synthesized by reacting 3-chloro-1-\{4-[3(substituted-phenyl)prop-2-enoyl]phenyl $\}$-4-(4-hydroxyphenyl)azetidin-2-one $\left(\begin{array}{lll}0.01 & \mathrm{M})\end{array}\right)$ and phenyl hydrazine $(0.01 \mathrm{M})$ in presence of acetic acid (glacial). All these compounds were characterized by means of their IR, ${ }^{1} \mathrm{H}$ NMR, spectroscopic data and microanalysis. All the synthesized products were evaluated for their antimicrobial activity. All the compounds were tested for their antibacterial and antifungal activities by broth dilution method.
\end{abstract}

Keywords: Chalcones, Phenyl pyrazolines, Azetidin-2-one, Antimicrobial activity

\section{Introduction}

Nitrogen and oxygen containing heterocycles play a predominant role in medicinal chemistry and they have been intensively used as scaffolds for drug development. Considerable attention has been focused on pyrazolines and substituted pyrazolines due to their interesting biological activities. To synthesize pyrazoline derivatives, we selected chalcone as starting material. Generally chalcones are 1,3- diaryl-2-propene-1-ones.

Some substituted pyrazolines and their derivatives have been reported to possess some interesting biological activities such as anticancer ${ }^{1}$, insecticidal ${ }^{2}$, antibacterial ${ }^{3}$, antifungal ${ }^{4}$, antidepressant $^{5-8}$, anticonvulsant ${ }^{9-10}$, anti-inflammatory ${ }^{11}$, antibacterial ${ }^{12}$ and antitumor ${ }^{13}$ properties. Moreover, many selectively fluoro-substituted organic compounds show peculiar pharmacological and agrochemical properties ${ }^{14-17}$. In the present study, the reaction of 3chloro-1-\{4-[3-(substituted phenyl) prop-2-enoyl] phenyl\}-4-(4-hydroxyphenyl) azetidin-2one with phenyl hydrazine in presence of acetic acid (glacial) to form pyrazoline derivatives 4a-j (Scheme 1). The structures of the various synthesized compounds were assigned on the basis of IR, ${ }^{1} \mathrm{H}$ NMR spectral data and elemental analysis. These compounds were also screened for their antimicrobial activity. 


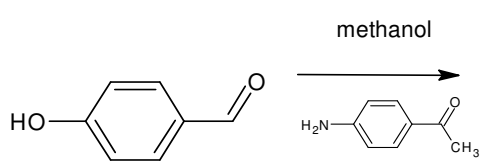<smiles>CC(=O)c1ccc(/N=C/c2ccc(O)cc2)cc1</smiles><smiles>CCCCCCCCC(=O)OCl</smiles><smiles>CC(=O)c1ccc(N2C(=O)C(Cl)C2c2ccc(O)cc2)cc1</smiles><smiles>O=C1C(Cl)C(c2ccc(O)cc2)N1c1ccc(C2=NN(c3ccccc3)C(C3C=CC=C3P)C2)cc1</smiles>

Scheme 1

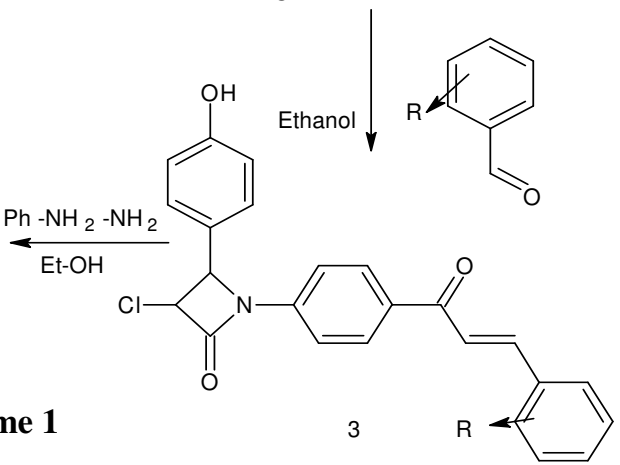

\section{Experimental}

The IR spectra were recorded on IR affinity-1, DRS-8000A, Shimadzu, Ptc. Ltd., Japan spectrophotometer. The ${ }^{1} \mathrm{H}-\mathrm{NMR}$ was recorded in DMSO on bruker advance II $400 \mathrm{MHz}$ spectrometer using TMS as an internal standard. Melting points were determined in open capillary tubes and are uncorrected (Table 1). The purity of the compounds was checked by TLC-using silica gel-G (Merck). Column chromatography was performed on silica gel.

Preparation of 1-(4-\{[(4-hydroxyphenyl) methylene] amino\} phenyl) ethanone (1)

A mixture of 4-hydroxy benzaldehyde $(0.01 \mathrm{M}), 1$-(4-aminophenyl) ethanone $(0.01 \mathrm{M})$ and methanol $(30 \mathrm{~mL})$ was heated for about $5 \mathrm{~min}$. in a beaker $(250 \mathrm{~mL})$ to get a clear solution. The solution was kept overnight at room temperature to get the respective crude solid which was recrystallized from ethanol to obtain the pure crystals of 1-(4-\{[(4-hydroxy phenyl) methylene]amino \}phenyl)ethanone respectively. The yield of the product was $75 \%$ and the product melts at $195{ }^{\circ} \mathrm{C}$. Found: $\mathrm{C}(75.28 \%) \mathrm{H}(5.45 \%) \mathrm{N}(5.82 \%)$ Calcd. for $\mathrm{C}_{15} \mathrm{H}_{13} \mathrm{NO}_{2}$ : $\mathrm{C}(75.30 \%) \quad \mathrm{H}(5.48 \%) \quad \mathrm{N}(5.85 \%) . \quad \mathrm{IR}, \quad \mathrm{cm}^{-1}: 3385 \quad(-\mathrm{OH}), \quad 3040(=\mathrm{C}-\mathrm{H}), \quad 2920(-\mathrm{C}-\mathrm{H})$, 1676( $>\mathrm{C}=\mathrm{O}), 1647(>\mathrm{C}=\mathrm{N}-), 1606(>\mathrm{C}=\mathrm{C}<), 1363\left(-\mathrm{CH}_{3}\right.$, bend $), 1314(-\mathrm{C}-\mathrm{N}<), 1284(-\mathrm{C}-\mathrm{O}-)$, 1240(-C-CO-C-). ${ }^{1} \mathrm{H}$ NMR (DMSO, $\left.\delta, \mathrm{ppm}\right): 2.5692\left(3 \mathrm{H}, \mathrm{s}, \mathrm{COCH}_{3}\right), 6.5277-7.9774(8 \mathrm{H}$, m, Ar-H), 8.3820 (1H, s, -CH=N-), 9.6392 ( 1H, s, Ar-OH). 
Preparation of 1-(4-acetylphenyl)-3-chloro-4-(4-hydroxyphenyl) azetidin-2-one (2)

In a $100 \mathrm{~mL}$ round bottom flask 1-(4-\{[(4-hydroxyphenyl) methylene] amino\} phenyl) ethanone $(0.01 \mathrm{M})$ in $70 \mathrm{~mL}$ benzene was taken. Chloro acetyl chloride $(0.01 \mathrm{M})$ was added at room temperature with constant stirring and triethylamine $1 \mathrm{~mL}$ was added and the reaction mixture was refluxed for 7 hours. After the completion of reaction, solvent was removed by vacuum distillation. The solid was filtered, dried and recrystallized from toluene. The yield of the product was $60 \%$ and the product melts at $119{ }^{\circ} \mathrm{C}$. Found: $\mathrm{C}(64.64 \%) \mathrm{H}(4.44 \%) \mathrm{N}(4.42 \%)$, Calcd. for $\mathrm{C}_{17} \mathrm{H}_{14} \mathrm{ClNO}_{3}$ : C $(64.67 \%) \mathrm{H}(4.47 \%) \mathrm{N}(4.44 \%)$. IR, $\mathrm{cm}^{-1}: 3300(-\mathrm{OH}), 3050(=\mathrm{C}-\mathrm{H}), 2950(-\mathrm{C}-\mathrm{H})$, 1680( $>\mathrm{C}=\mathrm{O}), 1600(>\mathrm{C}=\mathrm{C}<), 1375\left(-\mathrm{CH}_{3}\right.$, bend), 1300(-C-N<), 1240(-C-CO-C-), 1220 (-C-O-), 560(-C-Cl). ${ }^{1} \mathrm{H}$ NMR (DMSO, $\left.\delta, \mathrm{ppm}\right): 2.5392\left(3 \mathrm{H}, \mathrm{s}, \mathrm{COCH}_{3}\right), 4.8954(1 \mathrm{H}, \mathrm{d},>\mathrm{CH}-\mathrm{Ar})$, 5.5151 (1H, d, >CH-Cl), 6.6720-8.0745 (8H, m, Ar-H), 9.7784 (1H, s, Ar-OH).

Preparation of 3-chloro-1-\{4-[3-(Substituted phenyl) prop-2-enoyl] phenyl\}-4-(4hydroxyphenyl) azetidin-2-one (3)

To the solution of 1-(4-acetylphenyl)-3-chloro-4-(4-hydroxyphenyl) azetidin-2-one (0.01 M) in absolute ethanol $(50 \mathrm{~mL})$, substituted benzaldehyde $(0.01 \mathrm{M})$ and $2 \% \mathrm{NaOH}$ were added and refluxed for 10 hours. After refluxing the reaction mixture was concentrated, cooled, filtered and neutralized with dil. $\mathrm{HCl}$. The solid residue thus obtained was crystallized by absolute ethanol. IR(3b), $\mathrm{cm}^{-1}$ :3359(-OH), 3045(=C-H), 1728(>C=O), 1608(>C=C<), 1290(-C-N<), 1186 (-C-O-), 759(-C-Cl). ${ }^{1} \mathrm{H}$ NMR (3c-DMSO, $\delta$, ppm): $3.8739\left(6 \mathrm{H}, \mathrm{s},-\mathrm{OCH}_{3}\right), 4.8613(1 \mathrm{H}, \mathrm{d},>\mathrm{CH}-\mathrm{Ar}), 5.403$ (1H, d, >CH-Cl), 6.7340-7.8883 (11H, m, Ar-H), 7.9733 (2H, d, -CH=CH-), 9.8306 (1H, s, Ar-OH).

Preparation 4-(4-hydroxyphenyl)-3-chloro-1-\{4-[5-(Substituted phenyl)-1-phenyl-4, 5-dihydro-pyrazol-3-yl] phenyl\} azetidin-2-one (4a-j)

A mixture of 3-chloro-1-\{4-[3-(substituted phenyl) prop-2-enoyl] phenyl $\}$-4-(4hydroxyphenyl) azetidin-2-one $(0.01 \mathrm{M})$ and phenyl hydrazine $(0.01 \mathrm{M})$ in acetic acid $(30 \mathrm{~mL})$ was refluxed in an oil bath at $110-120{ }^{\circ} \mathrm{C}$ for 4 hours. The reaction mixture was poured over crushed ice; product was isolated and crystallized from ethanol.

IR (4a), cm ${ }^{-1}:$ 3362(-OH), 3041 $(=\mathrm{C}-\mathrm{H}), 1722(>\mathrm{C}=\mathrm{O}), 1624(>\mathrm{C}=\mathrm{N}), 1529(>\mathrm{C}=\mathrm{C}<)$, $1444\left(-\mathrm{CH}_{2}-\right), 1286(-\mathrm{C}-\mathrm{N}<), 1230(-\mathrm{C}-\mathrm{N}), 1173$ (-C-O-), 688(-C-Cl). ${ }^{1} \mathrm{H}$ NMR (4j-DMSO, $\delta$, ppm): $3.8689\left(3 \mathrm{H}, \mathrm{s}, \mathrm{OCH}_{3}\right), 3.6774\left(2 \mathrm{H}, \mathrm{d}, \mathrm{CH}_{2}\right.$ of Pyrazol), $4.3421(1 \mathrm{H}, \mathrm{t},>\mathrm{CH}-\mathrm{Ar}$ of Pyrazol), 4.8891 ( $1 \mathrm{H}, \mathrm{d},>\mathrm{CH}-\mathrm{Ar}$ of Azetidine), $5.2723(1 \mathrm{H}, \mathrm{d},>\mathrm{CH}-\mathrm{Cl}$ of Azetidine), 6.4416-7.9982 (16H, m, Ar-H, -NH-), 9.2940 (1H, s, Ar-OH).

\section{Results and Discussion}

Antimicrobial activity

The MICs of synthesized compounds were carried out by broth micro dilution method as described by Rattan ${ }^{18}$. It is one of the non automated in vitro bacterial susceptibility tests. This classic method yields a quantitative result for the amount of antimicrobial agents that is needed to inhibit growth of specific microorganisms.

The in vitro antimicrobial activity of test compounds were assessed against $24 \mathrm{~h}$ cultures of several selected bacteria and fungi. The bacteria used were E. coli, S.aureus, $P$. aeruginosa and S. pyogenus; the fungi used were C. albicans, A. Niger and A.clavatus.

The antimicrobial activity was performed by broth dilution method in DMSO. Gentamycin, Ampicilin, Chloramphenicol, Ciprofloxacin, Norfloxacin, Nystatin and Greseofulvin were used as standard for the evaluation of antibacterial and antifungal activities respectively. The activity was reported by minimal inhibition concentration. The results are summarized in Tables 2-4. 
Table 1. Physical constant of 4-(4-hydroxyphenyl)-3-chloro-1-\{4-[5-(substituted phenyl)-1phenyl-4, 5-dihydro-pyrazol-3-yl] phenyl\} azetidin-2-one (4a-j)

\begin{tabular}{|c|c|c|c|c|c|c|c|}
\hline \multirow[b]{2}{*}{ Compd } & \multirow[b]{2}{*}{$\mathrm{R}$} & \multirow[b]{2}{*}{ M.F. } & \multirow[b]{2}{*}{$\begin{array}{c}\text { Yield } \\
\%\end{array}$} & \multirow[b]{2}{*}{$\begin{array}{c}\text { M.P. } \\
{ }^{\circ} \mathrm{C}\end{array}$} & \multicolumn{3}{|c|}{ Elemental analysis } \\
\hline & & & & & $\begin{array}{c}\% \mathrm{C} \\
\text { Found } \\
\text { Calcd }\end{array}$ & $\begin{array}{l}\% \mathrm{~N} \\
\text { Found } \\
\text { Calcd }\end{array}$ & $\begin{array}{l}\% \mathrm{H} \\
\text { Found } \\
\text { Calcd }\end{array}$ \\
\hline \multirow{2}{*}{$4 a$} & \multirow{2}{*}{$-2-\mathrm{Cl}$} & \multirow{2}{*}{$\mathrm{C}_{30} \mathrm{H}_{23} \mathrm{Cl}_{2} \mathrm{~N}_{3} \mathrm{O}_{2}$} & \multirow{2}{*}{72} & \multirow{2}{*}{98} & 68.14 & 7.91 & 4.32 \\
\hline & & & & & 68.19 & 7.95 & 4.39 \\
\hline \multirow{2}{*}{$4 b$} & \multirow{2}{*}{$-2-\mathrm{OH}$} & \multirow{2}{*}{$\mathrm{C}_{30} \mathrm{H}_{24} \mathrm{ClN}_{3} \mathrm{O}_{3}$} & \multirow{2}{*}{74} & \multirow{2}{*}{174} & 70.61 & 8.20 & 4.70 \\
\hline & & & & & 70.65 & 8.24 & 4.74 \\
\hline \multirow{2}{*}{$4 c$} & \multirow{2}{*}{$\begin{array}{c}-3,4- \\
\left(\mathrm{OCH}_{3}\right)_{2}\end{array}$} & \multirow{2}{*}{$\mathrm{C}_{32} \mathrm{H}_{28} \mathrm{ClN}_{3} \mathrm{O}_{4}$} & \multirow{2}{*}{71} & \multirow{2}{*}{158} & 69.32 & 7.52 & 5.02 \\
\hline & & & & & 69.37 & 7.58 & 5.09 \\
\hline \multirow{2}{*}{ 4d } & \multirow{2}{*}{$-3-\mathrm{NO}_{2}$} & \multirow{2}{*}{$\mathrm{C}_{30} \mathrm{H}_{28} \mathrm{ClN}_{4} \mathrm{O}_{4}$} & \multirow{2}{*}{76} & \multirow{2}{*}{165} & 66.80 & 10.32 & 4.26 \\
\hline & & & & & 66.85 & 10.39 & 4.30 \\
\hline \multirow{2}{*}{$4 e$} & \multirow{2}{*}{$-4-\mathrm{Cl}$} & \multirow{2}{*}{$\mathrm{C}_{30} \mathrm{H}_{23} \mathrm{Cl}_{2} \mathrm{~N}_{3} \mathrm{O}_{2}$} & \multirow{2}{*}{69} & \multirow{2}{*}{164} & 68.15 & 7.89 & 4.32 \\
\hline & & & & & 68.19 & 7.95 & 4.39 \\
\hline \multirow{2}{*}{$4 f$} & \multirow{2}{*}{$\begin{array}{c}-4- \\
\mathrm{N}\left(\mathrm{C}_{2} \mathrm{H}_{5}\right)_{2}\end{array}$} & \multirow{2}{*}{$\mathrm{C}_{34} \mathrm{H}_{33} \mathrm{ClN}_{4} \mathrm{O}_{2}$} & 72 & 168 & 72.20 & 9.88 & 5.85 \\
\hline & & & 12 & 108 & 72.26 & 9.91 & 5.89 \\
\hline $4 \mathrm{~g}$ & $-4-\mathrm{OH}$ & $\mathrm{C}_{30} \mathrm{H}_{24} \mathrm{ClN}_{3} \mathrm{O}_{3}$ & 78 & 158 & 70.60 & 8.19 & 4.70 \\
\hline & TII & & 10 & & 70.65 & 8.24 & 4.74 \\
\hline 4h & $-4-$ & $\mathrm{C}_{32} \mathrm{H}_{-}$ & 70 & 152 & 71.51 & 10.39 & 5.39 \\
\hline 411 & $\mathrm{~N}\left(\mathrm{CH}_{3}\right)_{2}$ & $\mathrm{C}_{32} \mathrm{\Pi}_{29} \mathrm{CIN} \mathrm{N}_{4} \mathrm{U}_{2}$ & & & 71.57 & 10.43 & 5.44 \\
\hline $4 i$ & $\mathrm{CHO}$ & $\mathrm{C}_{30} \mathrm{H}_{2} \mathrm{ClN}_{3} \mathrm{O}_{2}$ & 79 & 195 & 72.89 & 8.48 & 4.86 \\
\hline 71 & C10 & $301-24=21-302$ & & 193 & 72.94 & 8.51 & 4.90 \\
\hline $4 \mathbf{j}$ & $-2-\mathrm{OH}-$ & $\mathrm{C}_{31} \mathrm{H}_{26} \mathrm{ClN}_{3} \mathrm{O}_{4}$ & 77 & 95 & 68.91 & 7.72 & 4.80 \\
\hline $\mathrm{TJ}$ & $3-\mathrm{OCH}_{3}$ & & & & 68.95 & 7.78 & 4.85 \\
\hline
\end{tabular}

Table 2. Antimicrobial activity of 4-(4-hydroxyphenyl)-3-chloro-1-\{4-[5-(substituted phenyl)-1-phenyl-4, 5-dihydro-pyrazol-3-yl] phenyl\} azetidin-2-one

\begin{tabular}{|c|c|c|c|c|c|c|c|c|}
\hline \multirow{3}{*}{ Compd } & \multirow{3}{*}{$\mathrm{R}$} & \multicolumn{2}{|c|}{$\begin{array}{c}\text { Antibacterial } \\
\text { Minimal } \\
\text { Concentration }\end{array}$} & \multicolumn{2}{|c|}{$\begin{array}{c}\text { Activity } \\
\text { Inhibition }\end{array}$} & \multicolumn{2}{|c|}{$\begin{array}{c}\text { Antifungal } \\
\text { Minimal } \\
\text { Concentration } \\
\end{array}$} & \multirow{2}{*}{ 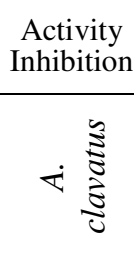 } \\
\hline & & 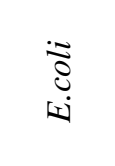 & R. & $\begin{array}{l}\tilde{\Xi} \\
\vdots \\
\vdots \\
\vdots \\
\dot{\Xi}\end{array}$ & 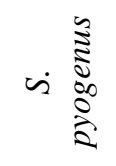 & ن $\frac{\mathfrak{\Xi}}{\stackrel{\tilde{\Xi}}{\tilde{\Xi}}}$ & 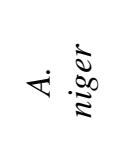 & \\
\hline & & $\begin{array}{c}\text { MTCC } \\
443\end{array}$ & $\begin{array}{c}\text { MTCC } \\
1688\end{array}$ & $\begin{array}{c}\text { MTCC } \\
96\end{array}$ & $\begin{array}{c}\text { MTCC } \\
442\end{array}$ & $\begin{array}{c}\text { MTCC } \\
227\end{array}$ & $\begin{array}{c}\text { MTCC } \\
282\end{array}$ & $\begin{array}{c}\text { MTCC } \\
1323\end{array}$ \\
\hline $4 a$ & $-2-\mathrm{Cl}$ & 100 & 62.5 & 100 & 100 & 250 & $>1000$ & $>1000$ \\
\hline $4 b$ & $-2-\mathrm{OH}$ & 175 & 200 & 175 & 250 & 800 & 700 & 700 \\
\hline $4 c$ & $\begin{array}{l}-3-\mathrm{OCH}_{3}, \\
-4-\mathrm{OCH}_{3}\end{array}$ & 225 & 225 & 150 & 200 & $>1000$ & 800 & 600 \\
\hline $4 d$ & $-3-\mathrm{NO}_{2}$ & 175 & 225 & 200 & 150 & 700 & 600 & 1000 \\
\hline $4 e$ & $-4-\mathrm{Cl}$ & 100 & 175 & 100 & 175 & 500 & $>1000$ & 1000 \\
\hline $4 f$ & $-4-\mathrm{N}\left(\mathrm{C}_{2} \mathrm{H}_{5}\right)_{2}$ & 125 & 200 & 100 & 100 & 250 & 1000 & 1000 \\
\hline $4 g$ & $-4-\mathrm{OH}$ & 200 & 250 & 62.5 & 125 & 500 & 800 & 800 \\
\hline $4 \mathrm{~h}$ & $-4-\mathrm{N}\left(\mathrm{CH}_{3}\right)_{2}$ & 200 & 250 & 125 & 200 & 250 & 1000 & 1000 \\
\hline $4 \mathbf{i}$ & $-\mathrm{H}$ & 175 & 225 & 200 & 125 & 750 & 600 & 800 \\
\hline $4 \mathbf{j}$ & $\begin{array}{c}-3-\mathrm{OCH}_{3}, \\
-4-\mathrm{OH}\end{array}$ & 200 & 200 & 225 & 200 & 1000 & 800 & $>1000$ \\
\hline
\end{tabular}


Table 3. Antibacterial activities- Minimal inhibition concentration (The standard drugs)

\begin{tabular}{ccccc}
\hline \multirow{2}{*}{ DRUG } & E.coli & P.aeruginosa & S.aureus & S.pyogenus \\
\cline { 2 - 5 } & MTCC 443 & MTCC 1688 & MTCC 96 & MTCC 442 \\
\hline (Microgramme/ML) & & & & \\
Gentamycin & 0.05 & 1 & 0.25 & 0.5 \\
Ampicillin & 100 & -- & 250 & 100 \\
Chloramphenicol & 50 & 50 & 50 & 50 \\
Ciprofloxacin & 25 & 25 & 50 & 50 \\
Norfloxacin & 10 & 10 & 10 & 10 \\
\hline
\end{tabular}

Table 4. Antifungal activity- Minimal inhibition concentration (The standard drugs)

\begin{tabular}{llll}
\hline DRUG & C.albicans & A.niger & A.clavatus \\
\hline- & MTCC 227 & MTCC 282 & MTCC 1323 \\
(Microgramme/ML) & & & \\
Nystatin & 100 & 100 & 100 \\
Greseofulvin & 500 & 100 & 100 \\
\hline
\end{tabular}

Biological screening result of 4-(4-hydroxyphenyl)-3-chloro-1-\{4-[5-(Substituted phenyl)-1-phenyl-4, 5-dihydro-pyrazol-3-yl] phenyl \} azetidin-2-one based derivatives shows that compound $\mathbf{4 a} \& \mathbf{4 e}$ have shown better activity against $E$. coli, while compound $\mathbf{4 a} \& \mathbf{4} \mathbf{f}$ have shown better activity against S. Pyogenus, while rest of all compound possessed good activity against S.aureus in the range of $100-225 \mu \mathrm{g} / \mathrm{mL}$ and $P$. Aeruginosa in the range $62.5-250 \mu \mathrm{g} / \mathrm{mL}$. Compound $\mathbf{4 e}$ and $\mathbf{4 g}$ is found to be significant antifungal activity against C. albicans, while rests of all derivatives are poor against $A$. niger and A.clavatus.

\section{Conclusion}

The main focus of this research work was to synthesize, characterize and evaluate antimicrobial activities of the newly synthesized phenyl pyrazoline derivatives, structures of synthesized compounds were confirmed and characterized with the help of analytical data's such as IR and ${ }^{1} \mathrm{H}-\mathrm{NMR}$. In summary, we have described the synthesis and antimicrobial activity of novel of 4-(4-hydroxyphenyl)-3-chloro-1-\{4-[5-(Substituted phenyl)-1-phenyl-4, 5-dihydro-pyrazol-3-yl] phenyl $\}$ azetidin-2-one MIC values revealed that amongst newly synthesized compound having chlorophenyl type linkage has shown good activity against the bacterial strains rest of all compounds exhibit moderate improvement in activity against some of the pathogenic strains.

\section{Acknowledgement}

The authors are thankful to the Principal and Management of Arts, Commerce \& Science College, Borsad and Mansa for providing laboratory facilities, SAIF, Chandigarh for NMR Spectra and Loyola Research Center- Xavier's College, Ahmedabad for IR spectra and micro-care laboratory, Surat, Gujarat, India for biological activity.

\section{References}

1. Hollis H D, Johnson J L, Werbel L M, Leopold W R, Jackson R C and Elslager E F, $J$ Med Chem., 1984, 27, 253.

2. Grosscurt A C, Van Hes R and Wellinga K, J Agric Food Chem., 1979, 27, 406-409.

3. Barot V M, Asian J Chem., 1996, 8(3), 565. 
4. Korgaokar S S, Patil P H, Shah M J and Parekh H H, Indian J Pharm Sci., 1996, 58, 222-225.

5. Palaska E, Aytemir M, Uzbay I T and Erol D, Eur J Med Chem., 2001, 36, 539-543.

6. Rajendra P Y, Lakshmana R A, Prasoona L, Murali K and Ravi K P, Bioorg Med Chem Lett., 2005, 15, 5030-5034.

7. Ozdemir Z, Kandilici H B, Gumusel B, Calis U and Bilgin A A, Eur J Med Chem., 2007, 42, 373-379.

8. Ruhogluo O, Ozdemir Z, Calis U, Gumusel B and Bilgin A A, Arzneimittelforschung, 2005, 55, 431-436.

9. Udupi R H, Kushnoor A S and Bhat A R, Indian J Heterocycl Chem., 1998, 8, 63-66.

10. Nauduri D and Reddy G B, Chem Pharm Bull (Tokyo), 1998, 46, 1254-1260.

11. Taylor E C and Patel H H, Tetrahedron, 1992, 48, 8089-8100.

12. Koga H, Itoh A, Murayama S, Suzue S and Irikura T, J Med Chem., 1980, 23(12), 1358-1363.

13. Wilkinson J A, Chem Rev., 1992, 92, 505-519.

14. Wise R, Andrews J M and Edwards L J, Antimicrb Agents Chemother., 1983, 23, 559-564.

15. Hagen S E, Domagala J M, Heifetz C L and Johnson J, J Med Chem., 1991, 34(3), 1155-1161.

16. Matsumoto J, Miyamoto T, Minamada A, Mishimuna Y, Egawa $\mathrm{H}$ and Nisimira $\mathrm{H}, J$ Med Chem., 1984, 27, 292-301.

17. Hayakawa I, Hiramitsu T and Tanaka Y, Chem Pharm Bull (Tokyo), 1984, 32(12), 4907-4913.

18. Rattan A, In: Antimicrobials in laboratory medicine, New Delhi, Churchill BI, Livingstone 2000, 85. 